\title{
The phosphorylation state of eNOS modulates vascular reactivity and outcome of cerebral ischemia in vivo
}

\author{
Dmitriy N. Atochin, ${ }^{1}$ Annie Wang, ${ }^{1}$ Victor W.T. Liu, ${ }^{1}$ Jeffrey D. Critchlow, ${ }^{1}$ Ana Paula V. Dantas, ${ }^{2}$ \\ Robin Looft-Wilson, ${ }^{3}$ Takahisa Murata, ${ }^{3}$ Salvatore Salomone, ${ }^{4}$ Hwa Kyoung Shin, ${ }^{4}$ Cenk Ayata, ${ }^{4}$ \\ Michael A. Moskowitz, ${ }^{4}$ Thomas Michel, ${ }^{2}$ William C. Sessa, ${ }^{3}$ and Paul L. Huang ${ }^{1}$ \\ ${ }^{1}$ Cardiovascular Research Center and Cardiology Division, Massachusetts General Hospital, Boston, Massachusetts, USA. ${ }^{2}$ Cardiovascular Division, \\ Brigham and Women's Hospital, Boston, Massachusetts, USA. ${ }^{3}$ Department of Pharmacology and Molecular Medicine, Yale University School of Medicine, \\ New Haven, Connecticut, USA. ${ }^{4}$ Stroke and Neurovascular Regulation Laboratory, Massachusetts General Hospital, Boston, Massachusetts, USA.
}

\begin{abstract}
NO plays critical roles in vascular function. We show that modulation of the eNOS serine 1179 (S1179) phosphorylation site affects vascular reactivity and determines stroke size in vivo. Transgenic mice expressing only a phosphomimetic (S1179D) form of eNOS show greater vascular reactivity, develop less severe strokes, and have improved cerebral blood flow in a middle cerebral artery occlusion model than mice expressing an unphosphorylatable (S1179A) form. These results provide a molecular mechanism by which multiple diverse cardiovascular risks, such as diabetes and obesity, may be centrally integrated by eNOS phosphorylation in vivo to influence blood flow and cardiovascular disease. They also demonstrate the in vivo relevance of posttranslational modification of eNOS in vascular function.
\end{abstract}

\section{Introduction}

eNOS plays important roles in vascular function, including regulation of vascular tone, angiogenesis, vascular signaling and remodeling, platelet aggregation, and leukocyte-endothelial interactions (1-4). eNOS activity is dependent on calcium-calmodulin activation. However, eNOS activity is also regulated at other levels, including availability of its substrate L-arginine or cofactors (NADPH, flavin adenine dinucleotide [FAD], flavin mononucleotide $[\mathrm{FMN}]$, and tetrahydrobiopterin $\left[\mathrm{BH}_{4}\right]$ ), protein-protein interactions with hsp90 and caveolins, posttranslational fatty acylation (myristoylation and palmitoylation), S-nitrosylation, and phosphorylation (5-7). Among these, eNOS phosphorylation appears to be an important means of regulating eNOS activity $(8,9)$.

eNOS phosphorylation at serine residue 1179 increases eNOS enzymatic activity and NO production. S1179 phosphorylation is increased by estrogens (10) and statins (11), accounting in part for the beneficial effects of these agents on vascular function. Shear stress and VEGF also increase S1179 phosphorylation (12). Leptin and the protective hormone adiponectin $(13,14)$ increase eNOS phosphorylation while IL-6, TNF- $\alpha$, and resistin decrease it (15, 16). eNOS phosphorylation provides a potential mechanistic link between metabolic abnormalities from diabetes or obesity and cardiovascular risk. We hypothesize that a defect in eNOS phosphorylation may account for endothelial dysfunction observed in hypertension, hyperlipidemia, diabetes, and metabolic syndrome. To date, the effects of eNOS phosphorylation on vascular function and disease pathogenesis have not been demonstrated in vivo.

The importance of eNOS phosphorylation at the $\mathrm{S} 1179$ residue has been studied using mutant forms of eNOS. S1179A eNOS

Nonstandard abbreviations used: $\mathrm{BH}_{4}$, tetrahydrobiopterin; $\mathrm{FAD}$, flavin adenine dinucleotide; FMN, flavin mononucleotide; L-NAME, $\mathrm{N}_{\omega}$-nitro-L-arginine methyl ester; MCA, middle cerebral artery; PSS, physiologic saline; SNP, sodium nitroprusside. Conflict of interest: The authors have declared that no conflict of interest exists. Citation for this article: J. Clin. Invest. 117:1961-1967 (2007). doi:10.1172/JCI29877. contains an alanine, which cannot be phosphorylated, in place of the serine. S1179D eNOS contains an aspartate with a carboxyl side group that mimics a negatively charged phosphate group in place of the serine. Purified S1179D eNOS enzyme has higher catalytic activity than WT eNOS due to both increased basal activity and calmodulin regulation of the reductase domain of eNOS (17). When these mutants are expressed in COS cells $(8,9)$ or endothelial cells (12), both S1179A and S1179D mutants are active. Thus, phosphorylation at $\mathrm{S} 1179$ is not a prerequisite for enzymatic activity, and the S1179A mutant is not a dominantnegative or null mutation. However, S1179A eNOS does not show Akt-dependent NO release while S1179D shows increased NO release even in the absence of Akt kinase $(8,9)$.

The molecular mechanism for the increased NOS activity in S1179D eNOS (and eNOS phosphorylated at S1179) appears to be enhanced electron flux through the reductase domain and reduced calmodulin dissociation (17). Elegant crystallographic studies show that the carboxyl tail of NOS normally retards electron transfer from the reductase domain by locking the FMN-binding domain into an electron-accepting position (18). Phosphorylation of S1179 results in a negative charge (as does substitution by aspartate in the S1179D mutation), inducing a conformational change that shifts the entire FMN domain to allow enhanced electron transfer through the reductase domain, activating the enzyme. As a result, S1179D eNOS produces NO at resting levels of intracellular calcium. Physiologically, shear stress, VEGF, and insulin activate eNOS because they promote eNOS S1179 phosphorylation. This allows greater eNOS activation to take place at any calcium concentration without substantially changing the affinity of calcium-activated calmodulin for eNOS.

To define the role of eNOS phosphorylation at S1179 in vascular function and disease pathogenesis in vivo, we generated transgenic mice that express either S1179A or S1179D eNOS. By breeding these animals with eNOS KO mice, we obtained mice that only express the S1179A and S1179D mutant forms of eNOS. We deter- 


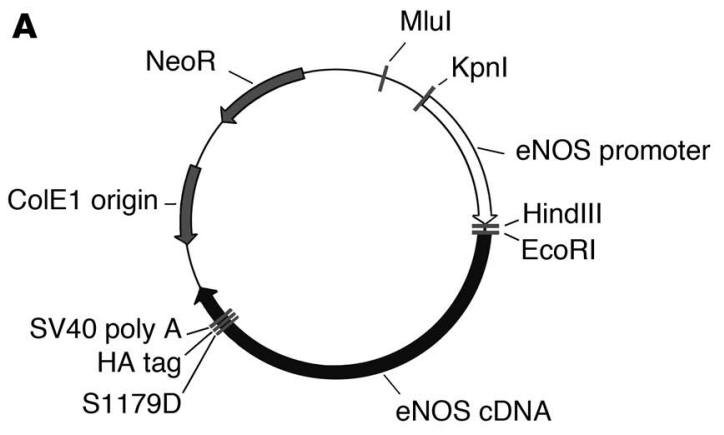

B

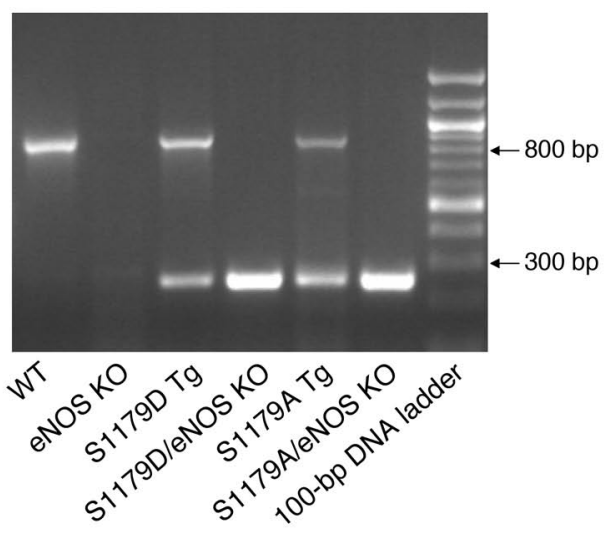

C

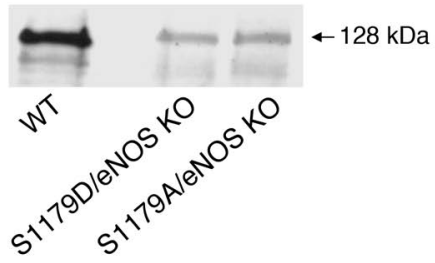

mined the effects of modulation of the S1179 phosphorylation site on vascular reactivity, cerebral blood flow, and outcome in a middle cerebral artery (MCA) occlusion model of stroke. Our results indicate that modulation of the S1179 phosphorylation site affects endothelium-dependent vasodilation and cerebral blood flow and that these effects determine outcome of stroke in vivo.

\section{Results}

Generation of S1179A and S1179D transgenic mice. We generated transgenic mice that express either S1179A or S1179D mutant forms of eNOS, using the construct shown in Figure 1A. We performed sitedirected mutagenesis on bovine eNOS cDNA to generate S1179A and S1179D mutations. The cDNAs were cloned into a vector containing an HA tag to facilitate detection of transgene expression. Expression of the eNOS cDNAs was driven using a 1.6-kb fragment of the human eNOS promoter, which is endothelium specific and contains the binding sites for Sp1, GATA-2, AP-1, NF-1, and the shear stress responsive element $(19,20)$. We established multiple independent founder lines for each mutant and selected 2 lines that showed equivalent levels of expression.

To obtain mice that express the mutant forms of eNOS but not endogenous WT eNOS, we bred the transgenic mice with eNOS KO mice (21) and selected offspring that carried the transgenes and were heterozygous for eNOS gene deletion. We then mated these animals with each other to obtain animals that carried the

\section{Figure 1}

Generation of S1179A and S1179D eNOS transgenic mice. (A) Transgenic construct. A 1.6-kb fragment of the human eNOS promoter drives the bovine eNOS CDNA carrying the S1179D or S1179A mutation, an HA tag, and the SV40 polyadenylation site. (B) Genotyping of transgenic and native eNOS genes by PCR. Primers E1 and E2 (see Methods) span an intron, so they amplify an 800 -bp product from the endogenous WT eNOS gene but a 280 -bp product from the S1179A and S1179D transgenes. Mice bred onto the homozygous eNOS KO background do not show the 800 -bp product. (C) Western blot analysis of heart protein $(60 \mu \mathrm{g})$ of WT, S1179D transgenic/eNOS KO (S1179D/ eNOS KO), and S1179A transgenic/eNOS KO (S1179A/eNOS KO) mice, using antibody directed against total eNOS.

transgenes and were homozygous for eNOS gene deletion. Thus, we obtained S1179D and S1179A transgenic mice on an eNOS null background (S1179D/eNOS KO mice and S1179A/eNOS KO mice). Figure 1B confirms the expression of the S1179A or S1179D transgene in the absence of the native eNOS gene.

The eNOS KO mice had been backcrossed to the C57BL/ 6 background, while the S1179A and S1179D transgenic mice were created on a C57BL/6-C3H F1 background. We tested the vascular responses of eNOS KO littermates from these matings that did not contain the S1179A or S1179D transgenes. These littermate control eNOS KO mice had vascular responses to ACh, sodium nitroprusside (SNP), and phenylephrine that were indistinguishable from de novo eNOS KO mice on a C57BL/6 background.

Expression and subcellular localization of S1179D and S1179A forms of eNOS. Western blot analysis confirmed that the expression levels of the mutant eNOS protein in S1179D and S1179A transgenic mice were equivalent but less than those seen in WT mice (Figure 1C). Consistent with this, the mean arterial blood pressure readings of S1179D/eNOS KO mice $(124 \pm 10 \mathrm{mmHg})$ and S1179A/ eNOS KO mice $(129 \pm 12 \mathrm{mmHg})$ were not significantly different from those of eNOS KO mice $(120 \pm 10 \mathrm{mmHg})$ while WT mice showed a mean arterial blood pressure of $90 \pm 10 \mathrm{mmHg}(n=3-5$ per group). Thus, we succeeded in reconstituting the eNOS KO mice with equivalent but diminished levels of either S1179D or S1179A forms of eNOS.

Figure 2 shows luminal en face vessel preparations stained by immunofluorescence. Expression of eNOS in the endothelial cells of WT mice is localized in a perinuclear Golgi pattern with lesser amounts of eNOS distributed throughout the cells. Expression of the mutant eNOS transgenes via immunostaining for the HA tag demonstrated that both S1179A and S1179D transgenes were expressed in a distribution similar to that observed for eNOS in WT cells, confirming proper subcellular localization of the proteins. Furthermore, staining for eNOS itself showed proper subcellular localization of the mutant forms of eNOS in the S1179D/ eNOS KO and S1179A/eNOS KO mice (Figure 3). The presence of the HA tag did not alter the subcellular localization of eNOS, nor did it affect the relationship between eNOS and caveolin-1, as seen in Figure 3.

Vascular reactivity in S1179A and S1179D mice. To determine how S1179 transgenes affect vascular reactivity, we mounted the carotid arteries onto a pressurized myograph, constricted the vessels with phenylephrine, and measured vascular relaxation in response to ACh. As seen in Figure 4A, S1179D/eNOS KO mice showed increased relaxation compared with S1179A/eNOS KO mice. eNOS KO mice without eNOS transgenes showed little relaxation. 
A

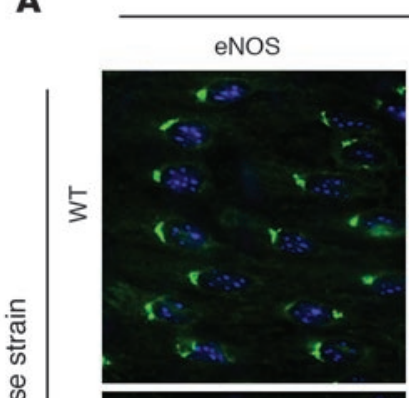

ฏ

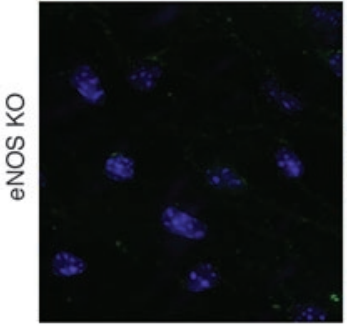

B

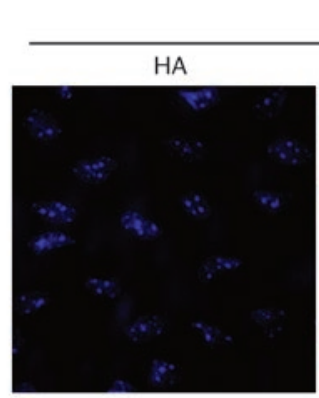

eNOS KO
Antibody
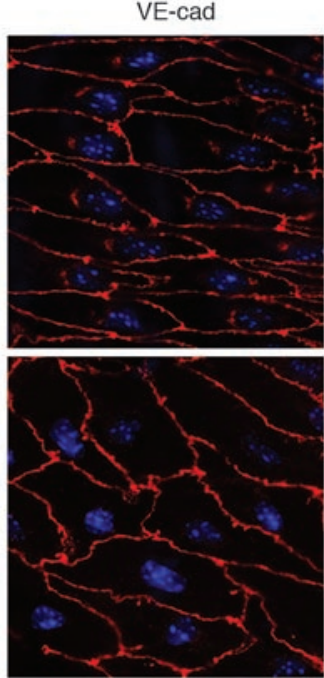

Antibody HA

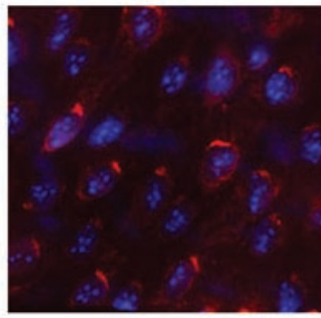

S1179A/eNOS KO

\section{eNOS and VE-cad}
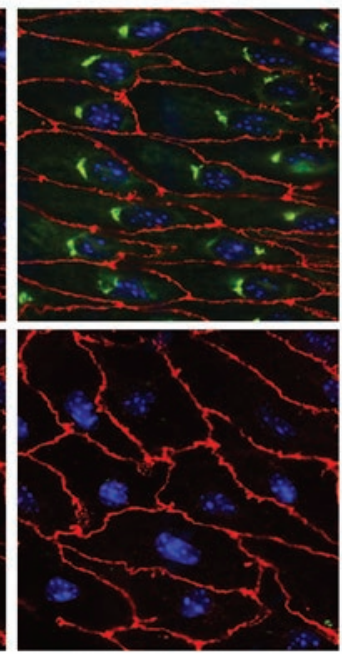

HA

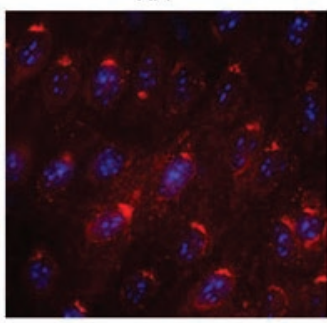

S1179D/eNOS KO

Mouse strain

\section{Figure 2}

Subcellular localization of WT and transgenic eNOS. (A) En face immunostaining of the common carotid arteries using eNOS antibody shows localization of eNOS in perinuclear Golgi that are aligned along the long axes of the cells in the endothelium. VE-cadherin (VE-cad) staining shows the outlines of the endothelial cell membranes. (B) En face immunostaining of the common carotid arteries using anti-HA shows perinuclear staining in the Golgi apparatus in a pattern similar to that of eNOS immunostaining in WT mice. Expression levels of the transgene are comparable in the S1179A/eNOS $\mathrm{KO}$ mice and S1179D/eNOS KO mice. Original magnification, $\times 600$.

residual blood flow calculated using a thresholding paradigm) compared with WT $(n=10)$ mice (Figure 6). These data show that eNOS activity is critical to augment blood flow in focal cerebral ischemia. Expression of the S1179A transgene in eNOS KO mice did not improve blood flow $(n=5)$ whereas expression of the S1179D eNOS transgene $(n=5)$ reduced the ischemic area to a size close to that seen in the WT mice.

\section{Discussion}

It is well established that hypertension, hyperlipidemia, diabetes, and smoking are associated with increased cardiovascular risk. However, the mechanisms by which these conditions increase atherosclerosis are not fully understood. One of the earliest detectable events in these conditions is endothelial dysfunction, which precedes structural changes. A relative insufficiency in vascular $\mathrm{NO}$ production results in a reduction in the protective

WT mice showed greater relaxation than S1179D/eNOS KO mice, consistent with the fact that the expression levels of mutant eNOS protein were less than WT expression levels. In contrast, carotid arteries from all 4 groups responded equally well to the endothelium-independent vasodilator SNP (Figure 4B).

MCA occlusion model of stroke. To determine the effect of these eNOS S1179 mutations on the response to cerebral ischemia, we subjected the S1179D/eNOS KO mice and S1179A/eNOS KO mice to an MCA occlusion model of stroke. Vessel occlusion for 1 hour followed by 23 hours of reperfusion resulted in a greater volume of cerebral infarction in eNOS KO mice compared with WT mice (22). Expression of the mutant S1179D eNOS transgene reduced infarct area while expression of S1179A eNOS did not (Figure 5). The functional significance of these results is confirmed by the neurologic scoring, which shows more severe deficits in the eNOS KO and S1179A/eNOS KO mice than in the WT and S1179D/eNOS KO mice.

Laser speckle flowmetry assessment of cerebral blood flow. To determine whether these differences in stroke size result from differences in cerebral blood flow, we performed laser speckle flowmetry during distal MCA occlusion (23). This technique measures cerebral blood flow with high spatial $(12 \mu \mathrm{m} /$ pixel $)$ and temporal resolution (1 image/7.5 s) and provides $2 \mathrm{D}$ assessment of blood flow deficit in real time. eNOS KO mice $(n=10)$ developed a significantly larger cortical area with more severe blood flow reduction $(\leq 20 \%$ effects of $\mathrm{NO}$ and increases vascular wall inflammation, thrombosis, and smooth muscle cell proliferation. We hypothesized that abnormalities in eNOS phosphorylation are an important common pathway that links diverse cardiovascular risks with endothelial dysfunction to increase propensity to atherosclerotic cardiovascular disease. For diabetes, obesity, and the metabolic syndrome, abnormalities in adipocyte signaling or metabolism may be the primary events that lead to downstream vascular abnormalities.

S1179 is phosphorylated not only by Akt kinase, but also by AMP kinase (24) and protein kinases A and G (25). In particular, insulin activates eNOS through Akt-mediated S1179 phosphorylation, thereby increasing blood flow and glucose uptake (26). In contrast, adiponectin, which is vasculoprotective, increases NO production not through Akt, but rather through AMP kinase-mediated S1179 phosphorylation (14). Thus, the phosphorylation state of S1179 may be a common integration point for multiple diverse signaling systems, such as insulin and adipokines. These results support the possibility that abnormalities in eNOS phosphorylation may underlie endothelial dysfunction from various causes.

Here, we test the hypothesis that phosphorylation at S1179 is an important determinant of vascular function and outcome of atherosclerotic disease. To date, the effects of S1179A and S1179D mutants have been characterized in cultured cells $(12,14,26)$ and using ex vivo gene transfer into isolated vessels $(12,27,28)$. Both S1179A and S1179D eNOS are enzymatically active, so S1179 


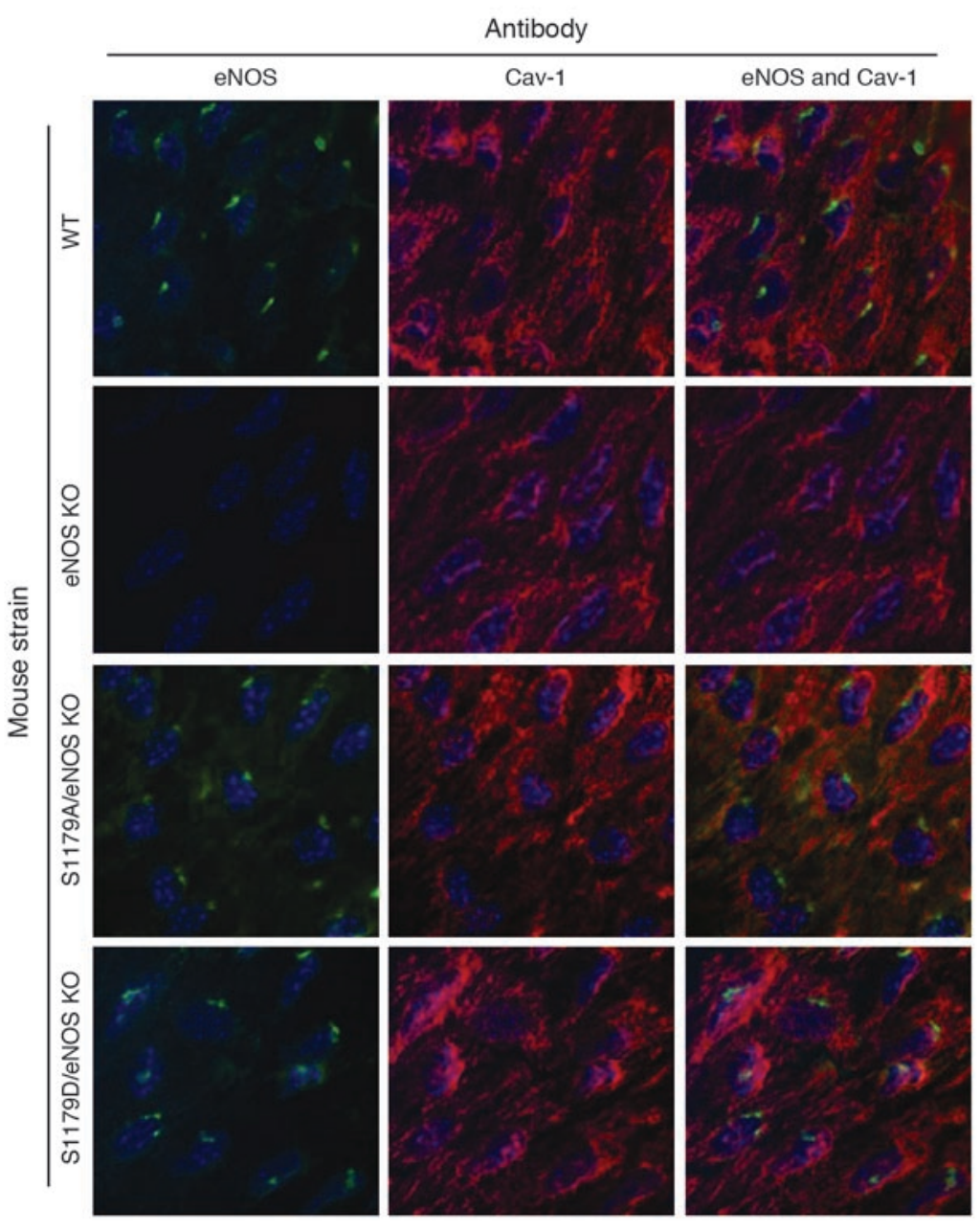

Figure 3

Interactions between caveolin-1 (Cav-1) and eNOS are unaffected in mice carrying transgenes. En face immunostaining of the common carotid arteries using eNOS antibody and caveolin-1 antibody shows that interactions between eNOS and caveolin-1 are unaffected in S1179D/eNOS KO mice and S1179A/eNOS KO mice. Original magnification, $\times 600$.

phosphorylation is not required for NO production. However, the $\mathrm{S1179D}$ form of eNOS is associated with much greater NO production due to enhanced electron flux through the reductase domain of eNOS and reduced calmodulin dissociation (17). As a result, $\mathrm{S} 1179 \mathrm{D}$ eNOS produces $\mathrm{NO}$ even in the absence of Akt kinase activity while S1179A fails to augment NO production in the setting of Akt activation $(8,9)$.

We find that S1179A, mimicking diminished S1179 phosphorylation, impairs vasodilation in intact vessels while the phosphomimetic S1179D eNOS increases vasodilation. Furthermore, modulation of the eNOS S1179 phosphorylation site affects cerebral blood flow in vivo and influences stroke size following cerebral ischemia. Because eNOS activity is regulated by multiple mechanisms, our results are important for demonstrating the in vivo relevance of this eNOS phosphorylation site to vascular function, blood flow, and outcome in a mouse model of human disease.

Although our results show that vascular reactivity and changes in blood flow are affected by eNOS phosphorylation at S1179, they do not preclude additional effects. Specifically, eNOS has been demonstrated to affect vascular smooth muscle proliferation, leukocyte-endothelial interactions, and platelet aggregation and adhesion. In addition, it is possible that eNOS phosphorylation and the resulting increase in NO production may also influence insulin sensitivity, as has been reported in eNOS homozygous and heterozygous $\mathrm{KO}$ mice $(29,30)$.

En face imaging of the HA tag shows that the human eNOS promoter (20) leads to expression in endothelial cells with preserved subcellular localization. Furthermore, Western blots show that expression levels of the S1179A and S1179D transgenes are equivalent, though diminished, compared with that of eNOS in WT mice. These considerations are important because differences in expression levels or localization could affect the outcome of vascular function studies or in vivo disease models. We compared S1179A/eNOS KO mice with S1179D/eNOS $\mathrm{KO}$ mice to directly assess the effects of phosphorylation on endothelial function, cerebral blood flow, and outcome of MCA occlusion. Comparisons with WT mice may be less revealing because the phosphorylation state of the S1179 residue in WT mice cannot be controlled and because the expression levels in the transgenic mice were less than WT eNOS levels. In this regard, reconstitution of eNOS KO mice with low levels of either S1179A or S1179D transgenes may explain why we did not observe compensatory effects of increased transgene expression that have been reported in some $(28)$ but not other $(12,27)$ systems.

eNOS KO mice have been used as a model of endothelial dysfunction because their phenotype reflects the effects of absence of endothelial NO production. However, most cardiovascular risks are not associated with total absence of vascular NO but rather a decrease in the amount of bioavailable NO. In particular, shear stress, insulin, IGF-1, estrogen, and VEGF influence eNOS activity through S1179 phosphorylation, so the S1179A and S1179D transgenic mice should be more pathophysiologically relevant models of endothelial dysfunction than mice that totally lack eNOS. Our results establish that eNOS phosphorylation is an important determinant of vascular function, blood flow, and outcome of cerebral ischemia in intact animals. This provides proof of concept for the modulation of the S1179 phosphorylation state as an approach to treating and preventing cardiovascular disease, particularly as influenced by risk factors of diabetes, obesity, metabolic syndrome, hyperlipidemia, and hypertension.

\section{Methods}

Generation of S1179D and S1179A eNOS mutant transgenic mice. To generate S1179A and S1179D transgenic mice, we cloned a 1.6-kb fragment (from $\mathrm{KpnI}$ to HindIII) containing the human eNOS promoter into pBK-CMV (Stratagene). Bovine eNOS cDNAs with HA tags and carrying either the S1179A or S1179D mutations were inserted into the EcoRI and BssHII restriction sites, placing the eNOS cDNAs in the correct orientation downstream of the human eNOS promoter. The plasmid map of the constructs is shown in Figure 1A. The plasmid inserts were liberated with MluI and NheI, purified, and injected into oocytes to obtain transgenic 
A

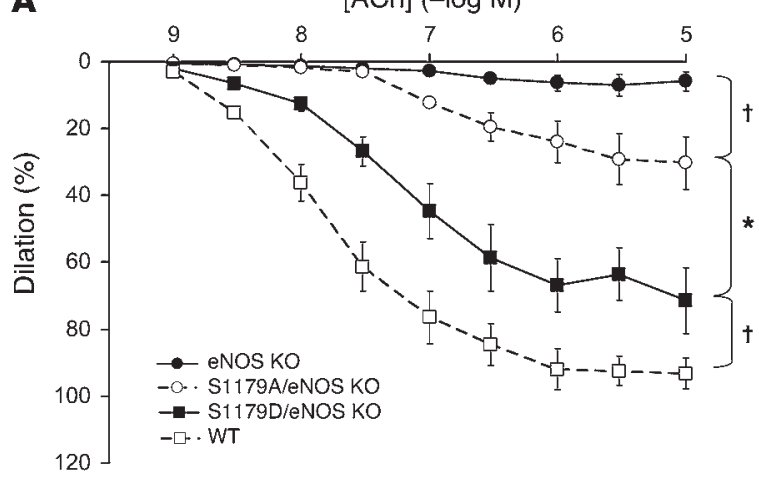

B

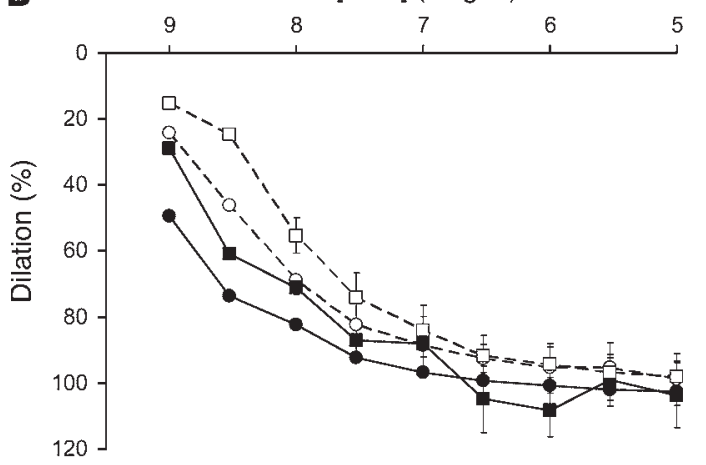

\section{Figure 4}

Effect of S1179D and S1179A mutations on vascular reactivity. (A) Left common carotid arteries were constricted with phenylephrine $\left(10^{-5} \mathrm{M}\right)$ and then subjected to increasing doses of ACh from $1 \times 10^{-9}$ to $1 \times 10^{-5} \mathrm{M}$. S1179A/eNOS KO mice, $n=8$; S1179D/eNOS KO mice, $n=8$; eNOS KO mice, $n=6$; and WT mice, $n=8 .{ }^{*} P<0.01$ by 1 -way ANOVA; ${ }^{\dagger} P<0.05$ by 1 -way ANOVA. (B) Left common carotid arteries were treated with L-NAME $\left(3 \times 10^{-4} \mathrm{M}\right)$ for 30 minutes, constricted with phenylephrine $\left(10^{-5} \mathrm{M}\right)$, and then subjected to increasing doses of SNP from $1 \times 10^{-9}$ to $1 \times 10^{-5} \mathrm{M}$. Data are expressed as mean \pm SEM.

Genotyping. Mice were genotyped using the following eNOS gene-specific primers: E1, 5'-GGGCTCCCTCCTTCCGGCTGCCACC-3'; and E2, 5'-GGATCCCTGGAAAAGGCGGTGAGG-3'. We used conditions of denaturation at $94^{\circ} \mathrm{C}$ for 30 seconds, annealing at $62^{\circ} \mathrm{C}$ for 60 seconds, and polymerization at $72^{\circ} \mathrm{C}$ for 60 seconds; 35 cycles. E1 and E2 span an intron, so they amplify an 800 -bp product (that includes the intron) from genomic DNA and a 280 -bp product (that does not include the intron) from the transgenes. All experiments were done on mice that express the S1179A or S1179D transgenes but not the endogenous WT eNOS gene. eNOS KO genotype was also confirmed by Southern blotting.

Western blot analysis. Heart tissue was homogenized to obtain protein extracts, and $60 \mu \mathrm{g}$ of protein was subjected to electrophoresis in a $7.5 \%$ Tris-HCl polyacrylamide gel. Proteins were transferred to membranes, incubated with a 1:250 dilution of primary mouse monoclonal anti-eNOS antibody (BD Biosciences), washed, incubated with 1:1,000 horseradish peroxidase-conjugated goat anti-mouse IgG secondary antibody, and visualized by chemiluminescence (Amersham Biosciences).

Blood pressure determination. Animals were anesthetized by inhalation using a mixture of $30 \% \mathrm{O}_{2}, 70 \% \mathrm{~N}_{2} \mathrm{O}$, and $1.5 \%$ isoflurane. Body temperature was maintained at $36-37^{\circ} \mathrm{C}$. The common carotid artery was exposed by dissection, and a catheter from pulled PE-10 tubing was inserted to measure systemic blood pressure using a fluid transducer (MacLab).

En face immunostaining. The common carotid artery was harvested, fixed with $4 \%$ paraformaldehyde in PBS at $4^{\circ} \mathrm{C}$ for 10 minutes, permeabilized with $0.3 \%$ Triton X-100 at room temperature for 30 minutes, and blocked with $3 \%$ fetal bovine serum at room temperature for 30 minutes. The secaccording to the recommendations of the American Veterinary Medical Association Panel on Euthanasia.

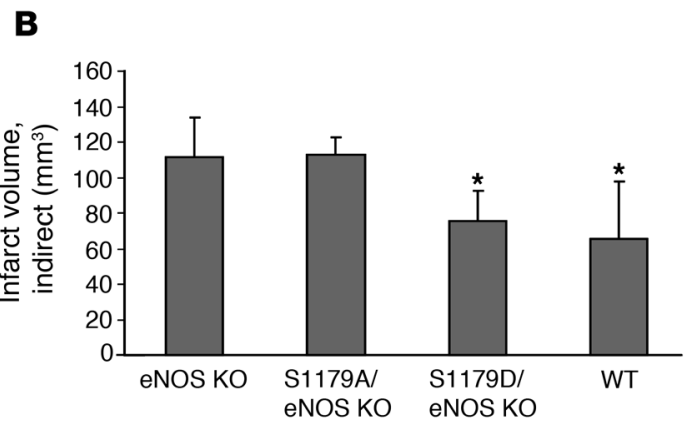

Figure 5

Effect of S1179A and S1179D eNOS mutations on cerebral infarct size. Mice were subjected to the filament model of MCA occlusion for 1 hour, followed by 23 hours of reperfusion. The brains were cut into 2-mm coronal sections and stained using 2,3,5-triphenyltetrazolium chloride. (A) Rostral to caudal distribution of infarct areas $\left(\mathrm{mm}^{2}\right)$ in coronal sections. Abscissa shows the distance $(\mathrm{mm})$ from the rostral surface of the brain. $n=7$ mice for each group. ${ }^{*} P<0.05$ versus corresponding sections from $\mathrm{S} 1179 \mathrm{~A} / \mathrm{eNOS}$ KO mice; ${ }^{\dagger} P<0.05$ versus corresponding sections from eNOS KO mice using 1-way ANOVA. (B) Infarct volumes were determined by integrating the infarct areas in each section over the entire brain, using the indirect method, which corrects for edema. ${ }^{*} P<0.05$ versus infarct volume from S1179A/eNOS and from eNOS KO mice using ANOVA. Neurologic scores for each group of mice are as follows: eNOS KO, 2.5; S1179A/eNOS KO, 2.67; S1179D/eNOS KO, 1.57; WT, 1.86. Kruskal-Wallis 1-way ANOVA on ranks showed a statistically significant difference between eNOS KO and S1179A/eNOS KO mice and WT and S1179D/eNOS KO mice $(P<0.05)$. 


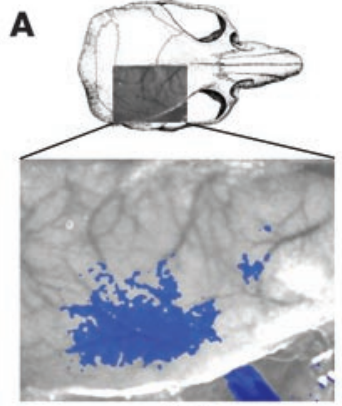

WT

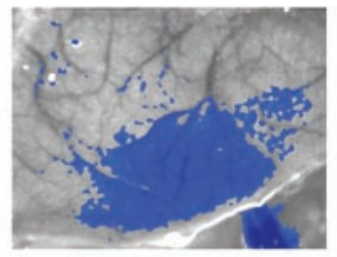

S1179A/eNOS KO

B

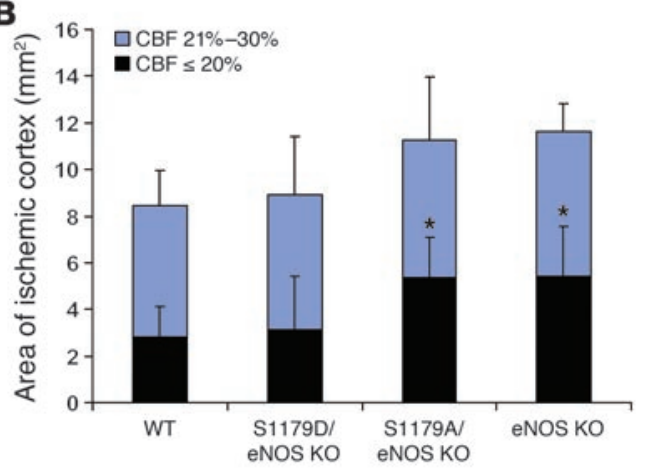

tions were incubated with mouse anti-eNOS antibody (BD Biosciences), rat anti-VE-cadherin antibody (BD Biosciences), rat anti-HA antibody (Roche Diagnostics), or rabbit anti-caveolin-1 antibody (Santa Cruz Biotechnology Inc.) at dilutions between 1:200 and 1:400 at $4^{\circ} \mathrm{C}$ for 12 hours. They were then incubated with a second antibody, either Alexa Fluor 488-conjugated goat anti-mouse IgG or Alexa Fluor 568-conjugated goat anti-rat IgG (Invitrogen) at 1:200 dilution at room temperature for 1 hour. The vessels were then visualized using a scanning microscope (Zeiss) with an Axiovert 200M imaging system.

Vascular reactivity studies. Phenylephrine, $\mathrm{ACh}, \mathrm{N}_{\omega}$-nitro-L-arginine methyl ester (L-NAME), and SNP were obtained from Sigma-Aldrich and dissolved in distilled water to make stock solutions. Mice were anesthetized with $30 \% \mathrm{O}_{2}, 70 \% \mathrm{~N}_{2} \mathrm{O}$, and $2 \%$ isoflurane. Carotid arteries were removed and mounted onto glass cannulas in a pressure myograph (DMT). The vessels were maintained at $85 \mathrm{mmHg}$ pressure and $37^{\circ} \mathrm{C}$. They were perfused in physiologic saline (PSS) with the following composition: $130 \mathrm{mM} \mathrm{NaCl}$, $4.7 \mathrm{mM} \mathrm{KCl}_{1} 1.17 \mathrm{mM} \mathrm{MgSO}_{4}, 14.9 \mathrm{mM} \mathrm{NaHCO}_{3}, 1.6 \mathrm{mM} \mathrm{CaCl}_{2}, 1.18$ $\mathrm{mM} \mathrm{KH}_{2} \mathrm{PO}_{4}, 0.026 \mathrm{mM}$ EDTA, and $5.5 \mathrm{mM}$ glucose. PSS was aerated at $95 \% \mathrm{O}_{2}$ and $5 \% \mathrm{CO}_{2}$ to maintain a $\mathrm{pH}$ of 7.4. The vessel diameter was continuously recorded using a video system controlled by custom software IonWizard (version 4.4; IonOptix Corp.). Vessels were equilibrated for 45 minutes and constricted with $10^{-5} \mathrm{M}$ phenylephrine. Response curves were then generated by stepwise application of increasing concentrations of ACh from $10^{-9}$ to $10^{-5} \mathrm{M}$ to the organ bath. The vessels were washed with PSS and equilibrated for 30 minutes. Basal NO production was inhibited by applying L-NAME $\left(3 \times 10^{-4} \mathrm{M}, 30\right.$ minutes $)$. Dose-response curves for

\section{Figure 6}

Effect of S1179A and S1179D eNOS mutations on cerebral blood flow. (A) Laser speckle contrast images in mice subjected to MCA occlusion. Superimposed areas (blue) indicate regions with $\leq 30 \%$ residual blood flow. Imaging field dimensions are $6 \times 8 \mathrm{~mm}$. Thresholded images were recorded after 60 minutes of ischemia. (B) Composite graph showing the area of cortex with $\leq 20 \%$ (black) or $21-30 \%$ (blue) residual blood flow compared with preischemic baseline. ${ }^{\star} P<0.05$, comparing the area with $\leq 20 \%$ of residual blood flow of eNOS KO and S1179A/eNOS KO mice versus WT and S1179D/eNOS KO mice. CBF, cerebral blood flow.

SNP were then generated by stepwise addition of increasing SNP concentrations from $10^{-9}$ to $10^{-5} \mathrm{M}$.

At the end of the experiments, passive diameters were determined by applying $\mathrm{Ca}^{2+}$-free PSS containing $2 \mathrm{mM}$ EGTA. ACh and SNP relaxation responses were expressed as percentage change in diameter after phenylephrine preconstriction compared with the difference between calciumfree diameter and diameter after phenylephrine constriction, using the following equation: percentage dilation $=100 \% \times\left[\left(D_{x}-D_{i}\right) /\left(D_{\text {Cafree }}-D_{i}\right)\right]$, where $D$ is the measured arterial diameter and subscripts $x, i$, and Ca-free denote arterial diameters at each dose of agonist $(x)$, initial diameter following phenylephrine constriction ( $i$ ), and in Ca-free buffer (Ca-free).

Filament model of MCA occlusion. For infarct volume, we used the filament model of MCA occlusion. Mice were anesthetized with $1.5 \%$ isoflurane in a mixture of $30 \% \mathrm{O}_{2}$ and $70 \% \mathrm{~N}_{2} \mathrm{O}$. Body temperature was maintained at $37^{\circ} \mathrm{C}$ using a thermostatic heating blanket (FHC). A flexible fiberoptic probe (Perimed) was affixed to the skull over the brain area supplied by the MCA (2 mm posterior and 6-7 mm lateral to bregma) for relative blood flow measurements by laser Doppler flowmetry. Baseline cerebral blood flow values were measured before internal carotid artery ligation and MCA occlusion and considered to be $100 \%$ flow. MCA occlusion was caused by inserting an 8-0 nylon filament (Doccol Corp.) covered by silicon into the internal carotid artery and advancing it to the origin of the MCA. Ischemia was confirmed by reduction in blood flow to less than $20 \%$ of control values, measured by laser Doppler flowmetry. Reperfusion was confirmed by Doppler after withdrawal of the filament.

Determination of infarct size. Infarct size was determined by staining with 2,3,5-triphenyltetrazolium chloride (TTC) (Sigma-Aldrich). Brains were cut into 2 -mm-thick coronal sections using a mouse brain matrix (RBM-200C; Activational Systems), stained with 2\% TTC for 1 hour at $37^{\circ} \mathrm{C}$ in the dark, and photographed with a digital camera. Sections were analyzed using an image analysis system (MCID M4; Imaging Research). Infarct sizes were determined by the indirect method, which corrects for edema (contralateral hemisphere volume minus volume of nonischemic ipsilateral hemisphere).

Neurologic scoring. Mice were examined for neurologic deficits 23 hours after MCA occlusion using a 5-point scale (31). Normal motor function was scored as 0 , flexion of the contralateral torso and forearm on lifting the animal by the tail as 1 , circling to the contralateral side but normal posture at rest as 2 , leaning to the contralateral side at rest as 3 , and no spontaneous motor activity as 4 .

Distal MCA occlusion. In order to perform laser speckle flowmetry throughout the entire ischemic period starting 5 minutes before its onset, we chose to occlude the MCA distally using a microvascular clip. Distal MCA occlusion can be performed during laser speckle flowmetry while the animal is on the stereotaxic frame and allows the imaging to continue uninterrupted. In contrast, filament occlusion of MCA requires the mouse to be in the supine position during insertion of the filament. Mice were anesthetized as above, intubated, paralyzed (pancuronium bromide, $0.4 \mathrm{mg} / \mathrm{kg}$ i.v. given every 45 
minutes), mechanically ventilated (model SAR-830 ventilator, CWE), and placed in a stereotaxic frame (Kopf). Arterial blood pressure, blood gases, and $\mathrm{pH}$ were monitored every 30 minutes. The temporalis muscle was separated from the temporal bone and removed. A burr hole ( $2 \mathrm{~mm}$ diameter) was drilled under saline cooling in the temporal bone overlying the MCA just above the zygomatic arch. The dura was kept intact, and the MCA was occluded using a microvascular clip (Zen clip; Oswa).

Laser speckle imaging of cerebral blood flow. For continuous monitoring of cerebral blood flow and ischemic area, a laser diode $(780 \mathrm{~nm})$ with a penetration depth of $500 \mu \mathrm{m}$ was used to illuminate the intact skull surface. Raw speckle images were acquired with a video camera (Cohu) and used to compute speckle contrast, a measure of speckle visibility related to the velocity of the scattering particles and therefore cerebral blood flow. Ten consecutive raw speckle images were acquired at $15 \mathrm{~Hz}$ and processed to speckle contrast using a sliding grid of $7 \times 7$ pixels. Contrast images were converted to correlation time values, and relative cerebral blood flow images were calculated. Perfusion images were obtained every 7.5 seconds starting before ischemia and continuing throughout the experiment. The areas of severe ( $0 \%-20 \%$ residual blood flow, representing core ischemic areas) and moderate (21\%-30\% residual blood flow, representing penumbra) blood flow deficits were quantified $\left(\mathrm{mm}^{2}\right)$ by using a thresholding paradigm as previously described (23).

1. Bredt, D.S., and Snyder, S.H. 1994. Nitric oxide: a physiologic messenger molecule. Annu. Rev. Biochem. 63:175-195.

2. Moncada, S., and Higgs, A. 1993. The L-argininenitric oxide pathway. N. Engl. J. Med. 329:2002-2012.

3. Dudzinski, D.M., Igarashi, J., Greif, D., and Michel, T. 2006. The regulation and pharmacology of endothelial nitric oxide synthase. Annu. Rev. Pharmacol. Toxicol. 46:235-276.

4. Moncada, S. 2006. Adventures in vascular biology: a tale of two mediators. Philos. Trans. R. Soc. Lond. B Biol. Sci. 361:735-759.

5. Alderton, W.K., Cooper, C.E., and Knowles, R.G. 2001. Nitric oxide synthases: structure, function and inhibition. Biochem. J. 357:593-615.

6. Huang, P.L. 2005. Unraveling the links between diabetes, obesity, and cardiovascular disease. Circ. Res. 96:1129-1131.

7. Shaul, P.W. 2002. Regulation of endothelial nitric oxide synthase: location, location, location. Annu. Rev. Physiol. 64:749-774.

8. Dimmeler, S., et al. 1999. Activation of nitric oxide synthase in endothelial cells by Akt-dependent phosphorylation. Nature. 399:601-605.

9. Fulton, D., et al. 1999. Regulation of endotheliumderived nitric oxide production by the protein kinase Akt. Nature. 399:597-601.

10. Hisamoto, K., et al. 2001. Estrogen induces the Aktdependent activation of endothelial nitric-oxide synthase in vascular endothelial cells. J. Biol. Chem. 276:3459-3467.

11. Kureishi, Y., et al. 2000. The HMG-CoA reductase inhibitor simvastatin activates the protein kinase Akt and promotes angiogenesis in normocholesterolemic animals. Nat. Med. 6:1004-1010.

12. Scotland, R.S., et al. 2002. Functional reconstitution of endothelial nitric oxide synthase reveals the importance of serine 1179 in endothelium-depen-

Statistics. All results are expressed as mean \pm SD. Responses to dilators were calculated as percentage reversal of induced tone or constriction. Statistical analysis was performed using 1-way ANOVA and post hoc test for infarct size. The area of cerebral blood flow deficit was analyzed using 2-way ANOVA for repeated measures. Statistical analysis for neurological deficit was performed using Kruskal-Wallis 1-way analysis of variance on ranks. Differences of $P<0.05$ were considered significant.

\section{Acknowledgments}

This work was supported by US Public Health Service grants NS10828, NS033335, HL057818, and HL048426 (to P.L. Huang).

Received for publication July 27, 2006, and accepted in revised form April 10, 2007.

Address correspondence to: Paul L. Huang, Cardiovascular Research Center, Massachusetts General Hospital, 149 Thirteenth Street, Room 4101, Charlestown, Massachusetts 02129, USA. Phone: (617) 724-9849; Fax: (617) 726-5806; E-mail: phuang1@partners.org.

Dmitriy N. Atochin and Annie Wang contributed equally to this work.

dent vasomotion. Circ. Res. 90:904-910.

13. Vecchione, C., et al. 2002. Leptin effect on endothelial nitric oxide is mediated through Aktendothelial nitric oxide synthase phosphorylation pathway. Diabetes. 51:168-173.

14. Chen, H., Montagnani, M., Funahashi, T., Shimomura, I., and Quon, M.J. 2003. Adiponectin stimulates production of nitric oxide in vascular endothelial cells. J. Biol. Chem. 278:45021-45026.

15. Shen, Y.H., et al. 2006. Up-regulation of PTEN (phosphatase and tensin homolog deleted on chromosome ten) mediates p38 MAPK stress signalinduced inhibition of insulin signaling. A crosstalk between stress signaling and insulin signaling in resistin-treated human endothelial cells. J. Biol. Chem. 281:7727-7736.

16. Kim, F., Gallis, B., and Corson, M.A. 2001. TNFalpha inhibits flow and insulin signaling leading to NO production in aortic endothelial cells. Am. J. Physiol. Cell Physiol. 280:C1057-C1065.

17. McCabe, T.J., Fulton, D., Roman, L.J., and Sessa, W.C. 2000. Enhanced electron flux and reduced calmodulin dissociation may explain "calciumindependent" eNOS activation by phosphorylation. J. Biol. Chem. 275:6123-6128.

18. Garcin, E.D., et al. 2004. Structural basis for isozymespecific regulation of electron transfer in nitric-oxide synthase. J. Biol. Chem. 279:37918-37927.

19. Robinson, L.J., Weremowicz, S., Morton, C.C., and Michel, T. 1994. Isolation and chromosomal localization of the human endothelial nitric oxide synthase (NOS3) gene. Genomics. 19:350-357.

20. Guillot, P.V., et al. 1999. A vascular bed-specific pathway. J. Clin. Invest. 103:799-805.

21. Huang, P.L., et al. 1995. Hypertension in mice lacking the gene for endothelial nitric oxide synthase. Nature. 377:239-242.

22. Huang, Z., et al. 1996. Enlarged infarcts in endothelial nitric oxide synthase knockout mice are attenuated by nitro-L-arginine. J. Cereb. Blood Flow Metab. 16:981-987.

23. Ayata, C., et al. 2004. Laser speckle flowmetry for the study of cerebrovascular physiology in normal and ischemic mouse cortex. J. Cereb. Blood Flow Metab. 24:744-755.

24. Chen, Z.P., et al. 1999. AMP-activated protein kinase phosphorylation of endothelial NO synthase. FEBS Lett. 443:285-289.

25. Butt, E., et al. 2000. Endothelial nitric-oxide synthase (type III) is activated and becomes calcium independent upon phosphorylation by cyclic nucleotide-dependent protein kinases. J. Biol. Chem. 275:5179-5187.

26. Montagnani, M., Chen, H., Barr, V.A., and Quon, M.J. 2001. Insulin-stimulated activation of eNOS is independent of $\mathrm{Ca} 2+$ but requires phosphorylation by Akt at Ser (1179). J. Biol. Chem. 276:30392-30398.

27. Akiyama, M., et al. 2002. Expression and function of recombinant S1179D endothelial nitric oxide synthase in canine cerebral arteries. Stroke. 33:1071-1076.

28. Sorenson, J., et al. 2005. Expression and function of recombinant S1179D endothelial NO synthase in human pial arteries. Stroke. 36:158-160.

29. Cook, S., et al. 2004. Partial gene deletion of endothelial nitric oxide synthase predisposes to exaggerated high-fat diet-induced insulin resistance and arterial hypertension. Diabetes. 53:2067-2072.

30. Cook, S., et al. 2003. Clustering of cardiovascular risk factors mimicking the human metabolic syndrome $\mathrm{X}$ in eNOS null mice. Swiss Med. Whly. 133:360-363.

31. Huang, Z., et al. 1994. Effects of cerebral ischemia in mice deficient in neuronal nitric oxide synthase. Science. 265:1883-1885. 